\title{
Comparación de la Politerapia vs Monoterapia antibiótica en la aplicación empírica en contra de las infecciones odontogénicas
}

\author{
David Alejandro Mendieta Rostrán. \\ Facultad de Medicina.
}

\section{RESUMEN}

Recibido: 29 de abril 2013 - Aprobado: 16 de julio 2016

El presente estudio se realizó con el objetivo de conocer la eficacia de las distintas terapéuticas de tratamiento contra las infecciones odontogénicas que se aplican a los pacientes ingresados con severidad moderada en el hospital Roberto Calderón los resultados obtenidos mostraron una similitud en la aplicación de la politerapia y la cobertura de manejo de la monoterapia. Por otro lado existe una diferencia significativa para este estudio ya que la politerapia presenta factores de evaluación desfavorable para esta terapia. Así mismo el manejo con monoterapia facilitó factores favorables en beneficio de este tratamiento ante las infecciones odontogénicas. A partir de los resultados descritos, esta investigación concluye que el manejo de las infecciones odontogénicas puede llevarse a cabo adecuadamente a nivel hospitalario por los pacientes afectados con severidad moderada por estas infecciones con la monoterapia de Clindamicina. Dando respuesta a la problemática planteada. Por tanto con este estudio se recomienda al servicio de cirugía oral y maxilofacial del Hospital escuela Roberto Calderón G. la creación de un protocolo para infecciones odontogénicas donde contenga en sus indicaciones el uso de la Monoterapia para pacientes con esta patología

Palabras clave: infecciones odontogénicas, politerapia, monoterapia, cirugía oral, Hospital escuela Roberto Calderón.

\section{SUMMARY}

The present study was conducted with the objective of knowing the efficacy of the different treatment therapies against odontogenic infections that are applied to patients admitted with moderate severity in the Roberto Calderón hospital. The results obtained showed a similarity in the application Of polytherapy and management coverage of monotherapy. On the other hand there is a significant difference for this study since the combination therapy presents unfavorable evaluation factors for this therapy. Likewise, monotherapy management facilitated favorable factors in favor of this treatment against odontogenic infections. From the results described, this research concludes that the management of odontogenic infections can be carried out adequately at the hospital level by patients affected with moderate severity from these infections with Clindamycin monotherapy. Responding to the problem raised. Therefore, with this study, it is recommended to the Oral and Maxillofacial Surgery Service of the Roberto Calderón G. School Hospital the creation of a protocol for odontogenic infections where it contains in its indications the use of Monotherapy for patients with this pathology

Key words: odontogenic infections, polytherapy, monotherapy, oral surgery, Roberto Calderón school hospital 


\section{INTRODUCCIÓN}

Los procesos infecciosos de origen odontogénico representan un problema enorme de salud pública por su marcada frecuencia dentro de la población y por ser motivo constante del uso empírico de los antibióticos en la prescripción médica. Siendo una de las patologías que con más frecuencia se atienden por el servicio de maxilofacial en el ámbito hospitalario en nuestro país. Esta patología afecta a toda la población desde edades infantiles hasta final de la vida lo que conlleva a un impacto considerable sobre la población y la economía destinada al tratamiento de estos pacientes.

Aunque las infecciones odontogénicas han venido declinando en las últimas décadas tanto en su incidencia, severidad y morbilidad - mortalidad. Dado esto a los estudios científicos acerca de la flora bacteriana patógena presente en la zona afectada por la infección en la región, Así como en la forma de contrarrestar la diseminación a los espacios anatómicos de la región facial y a los avances en las técnicas diagnósticas. Sigue siendo una patología difícil de manejar por los diversos criterios de los clínicos interesados del tema y la forma de abordar estas infecciones que proponen cada uno de ellos.

En la actualidad el manejo de las infecciones ontogénicas está basado en el control etiológico de origen de la patología, tratamiento precoz quirúrgico y el manejo sistémico de soporte (medidas físicas, hidratación, analgésicos), con el apoyo de un agente bacteriano de amplio espectro dado que esta patología es polimicrobiana. Se lleva a cabo este estudio con el objeto de comprobar la eficacia de las terapéuticas para el control y la erradicación de las infecciones odontogénicas en los pacientes que se ingresan al hospital Roberto Calderón Gutiérrez (HERCG). Con la intensión de crear un consenso médico para el buen manejo hospitalario de esta patología. Dando un buen uso de los fármacos antibióticos y aplicando debidamente la terapéutica.

\section{MATERIALES Y MÉTODO}

El tipo de estudio es descriptivo, analítico y no experimental. El área de estudio correspondió al servicio de cirugía oral y maxilofacial del Hospital Escuela Roberto Calderón Gutiérrez (HERCG), Managua, Nicaragua. La unidad de análisis fueron los pacientes hospitalizados con procesos infecciosos en los cuales el abordaje terapéutico se conllevó el uso de politerapia con ceftriazona clindamicina y los tratados con monoterapia de clindamicina. El universo fue de 136 pacientes que ingresaron en el servicio de cirugía oral y maxilofacial del centro hospitalario Roberto Calderón Gutiérrez entre el período del 2010 al 2013 con infecciones odontogénicas a los cuales se les aplicó la 
terapéutica para la resolución de dicha patología. Se tomaran a 40 pacientes, de los cuales 20 cumplirán con el tratamiento con politerapia y 20 pacientes con monoterapia antibiótica. Muestra efectuada a conveniencia para extraer datos equivalentes entre ambos grupos

Criterios de inclusión: pacientes con criterios de hospitalización por infecciones odontogénicas; consentimiento del paciente para ser tratado hospitalariamente por infecciones odontogénicas; pacientes en quienes se utilizó en la terapéutica de tratamiento a las infecciones odontogénicas, antibioticoterapia empírica; los pacientes que se les administró monoterapia con clindamicina y politerapia con clindamicina de manera empírica; los pacientes clasificados en grado 2 de severidad según la escala de Flynn.

Criterios de Exclusión: pacientes que no aceptaron el tratamiento hospitalario para infecciones de origen odontogénico; los pacientes clasificados en grado 1,3 y 4 según el store de severidad empleado por Flynn; pacientes que se presentaron al tratamiento con resultados de cultivo de secreciones y antibiograma para las infecciones odontogénicas; los pacientes que se trataron por infecciones odontogénicas con monoterapia que no se usó clindamicina y pacientes que aplicaron politerapia donde no se usó el esquema ceftriazona-clindamicina; pacientes con algún grado de inmunosupresión como: Diabetes, esteroides, inmunoterapia, quimioterapia, radioterapia, antibióticos de amplio espectro recurrentes, hospitalizaciones recurrentes, mal nutrición.

\section{RESULTADOS}

\section{Características sociodemográficas y datos de ingreso de los pacientes hospitalizados por} infecciones odontogénicas

La edad de los pacientes ingresados al HERCG con infecciones odontegénicas presentó el predomino entre la segunda y tercera etapa de la vida, pero se encontró pacientes en edad temprana y además hubo paciente por encima de los cincuenta años. La asociación de las infecciones odontogénicas según el sexo del paciente, el 58\% correspondió a las mujeres y con un mínimo de diferencia se establecieron los varones que alcanzaron el $42 \%$ de los 40 pacientes del estudio. El análisis descriptivo sobre la procedencia de los pacientes que se atendieron hospitalariamente por infecciones odontogénicas. Indica que el $73 \%$ son urbanos, el $20 \%$ son de procedencia rural y el restante $7,5 \%$ de los pacientes son de fuera de la ciudad. La escolaridad que presentaron los pacientes de este estudio evidencia que un $25 \%$ eran personas que tenían un nivel de escolaridad de secundaria 
completa y con el mismo porcentaje los que no han completado su secundaria. Mientras tanto, con un $22 \%$ se encontró a los que poseen una primaria completa, pero los que no han completado su primaria correspondieron al 28\% del total de pacientes que requirió el estudio. La afección de los espacios anatómicos que con mayor frecuencia se encontró fue en primer lugar, el espacio Submandibular con el 83\% de los pacientes valorados en el estudio. En segundo lugar de afección fue el espacio Mentoniano, que ocupo el 52\% de los pacientes. En un 15\% de los pacientes estaba afectado el espacio bucal. El $12 \%$ de los casos fue afectado el espacio sublingual y solo en un 5\% se afectó el espacio pterigomandibular.

En cuanto a los síntomas asociados a las infecciones odontogénicas al ingreso de cada paciente, se observa que el dolor estaba presente en el 100\% de los casos estudiados y que el $68 \%$ de los casos se presentaron con fiebre y la dificultad respiratoria o disnea, se presentó solo en un 10\%. Por la acción nociva propia de la infección odontogénica a los tejidos bucales incluyendo músculos masticadores, el trismo está presente en el $32 \%$ de los pacientes de gravedad moderada y con una afección leve por trismo correspondieron un 25\%. Sin embragó los pacientes que presentaron con una adecuada apertura oral fueron el $43 \%$ de los casos reportados en el estudio. El conteo de glóbulos blancos al ingreso hospitalario de los pacientes de este estudio demostró que la tendencia en los casos presentan leucocitosis por encima de los 10 x 10*9/L glóbulos blancos.

\section{Eficacia del tratamiento con politerapia empleado en la terapéutica en contra de las infecciones odontogénicas}

Los casos tratados con politerapia se les aplicó en un 100\% la terapéutica indicada para el tratamiento de las infecciones odontogénicas. Que constaba de la eliminación del factor etiológico, tratamiento quirúrgico precoz y apoyo con tratamiento de soporte sistémico más antibioticoterapia. La antibioticoterapia empleada fue Ceftriazona y Clindamicina. La vía de administración que se utilizó para la politerapia, da como resultado en este estudio, que en el 100\% de los casos se administra por vía intravenosa. El uso de las terapias intramusculares y vía oral no se efectúa en ninguno de los pacientes del estudio. La frecuencia con que se administró el antibiótico Ceftriazona en la politerapia fue una vez en el día con el 100\%. Mientras la aplicación de Clindamicina en la politerapia fue realizada cada 8hrs en el $100 \%$ de los pacientes que incluyen en el estudio. En la dosis aplicada en el tratamiento de politerapia con Ceftriazona se observa en la descripción de los resultados que dieron en un 100\% de los 
casos del estudio el uso de dos gramos de Ceftriazona. La dosis de 900mg de clindamicina se aplicó en el $100 \%$ de los pacientes. Mientras las dosis 600mg y 300mg no fueron aplicadas a los pacientes del estudio. Los efectos adversos de los fármacos de la politerapia, revelo que las náuseas fue el efecto más común con $25 \%$. Los mareos y trastornos gastrointestinales son los siguientes efectos que presenta en los pacientes el $15 \%$ y $12 \%$ respectivamente. A las reacciones adversas de Rash cutáneo y vomito no se presentaron en los pacientes de estudio. La valoración de cada paciente a las $48 \mathrm{hrs}$ de hospitalizados presentó que la presencia de dolor es el síntoma más frecuente en los pacientes de estudio ingresados, con un 45\%. La fiebre y la disnea no tuvieron datos que los asocio cuando se realizó el control en $48 \mathrm{hrs}$ de aplicación del tratamiento con politerapia en cada paciente.

El trismo presente a las $48 \mathrm{hrs}$ en la evaluación clínica de los pacientes dio como resultado el $75 \%$ de los casos presenta una adecuada apertura oral y el 25\% de los pacientes poseen una leve presencia de trismo en las $48 \mathrm{hrs} \mathrm{de} \mathrm{control.} \mathrm{En} \mathrm{cuanto} \mathrm{al} \mathrm{control} \mathrm{de} \mathrm{glóbulos} \mathrm{blancos} \mathrm{a} \mathrm{las} 48 \mathrm{hrs} \mathrm{de}$ aplicación de la terapéutica contra las infecciones odontogénicas, se observan evidencias de que en todos los casos hubo descenso en el conteo de glóbulos blancos. Con cifras por debajo de los 10x $10^{\wedge} 9 / \mathrm{L}$ en los reportes que se encuentran descritos en los exámenes de laboratorio llegando en alguno de los casos a valores aceptables de leucocitos. Los pacientes tratados con la terapéutica contra infecciones odontogénicas con politerapia, lo que mostró un 50\% de los pacientes tiene una estancia hospitalaria de 3 a 5 días, los pacientes que están en la categoría de 6 a 10 días de estancia hospitalaria corresponden al $30 \%$. Mientras los pacientes que poseen estancias hospitalarias de 11 a 15 son el $15 \%$ y que con $5 \%$ se presentaron los pacientes con más de 15 días de estancia hospitalaria.

Eficacia en el tratamiento con monoterapia en la terapéutica encontra de las infecciones de origen odontogénico

A los pacientes tratados con politerapia, se les aplicó en este estudio la terapéutica indicada para el tratamiento de las infecciones odontogénicas en un 100\% de los casos. Esta terapéutica igual que en la politerapia consta de la eliminación del factor etiológico, tratamiento quirúrgico precoz y apoyo con tratamiento de soporte sistémico más anibioticoterapia. La vía de administración intravenosa que se utilizó en la monoterapia fue en un 100\% de los casos. Mientras el uso de la aplicación de la terapia a través de la vía intramusculares y vía oral no se implementó en los pacientes de estudio. La frecuencia con que se aplicó la monoterapia con Clindamicina fue $100 \%$ en el intervalo de cada 8 hrs. Por otra parte los otros dos intervalos de aplicación cada $12 \mathrm{hrs}$ y una vez al día. No presentan datos en el 
estudio. Además, se representa en el $100 \%$ de los casos tratados con monoterapia que la dosis utilizada es 900mg de Clindamicina. Sin embargo no se contó con datos en el estudio de aplicación de $600 \mathrm{mg}$ de Clindamicina al igual que con 300mg de Clindamicina.

El dato correspondiente al dolor de mayor frecuencia al momento de realizar el control de los pacientes con $48 \mathrm{hrs}$ de ingreso hospitalarios manejados con monoterapia. Representando el dolor un $100 \%$. Por otro lado los síntomas fiebre y disnea no se presentan en los casos estudiados. Por otro lado, se muestra que $70 \%$ de los pacientes valorados en $48 \mathrm{hrs}$ de aplicación de la monoterapia presenta una adecuada apertura oral en los pacientes hospitalizados y el 30\% presentaban una leve presencia de trismo. Los glóbulos blancos que se obtuvieron en el control de laboratorio a las 48hrs de aplicación de Clindamicina contra los datos que se muestra en los pacientes al ingreso hospitalario da como resultado la disminución de glóbulos blancos por debajo de los 10x10^9/L en los casos del estudio manejados con monoterapia. Se muestra que en los días de estancia hospitalaria de los pacientes ingresados con tratamiento de monoterapia. El 75\% fue de 3 a 5 días y el 25\% de los casos ingresados están con 6 a 10 días de estancia.

Eficacia de cada terapéutica aplicada a los pacientes hospitalizados con infecciones odontogénicas de este estudio.

Los síntomas asociados a la infección odontogénica, en ambos grupos de pacientes del presente estudio, poseen similitud en sus datos con iguales porcentajes tanto en los síntomas: Dolor $100 \%$, fiebre $67.5 \%$ así como en la Disnea con 10\%. Además, en ambas terapéuticas el Dolor es el único síntoma que se presenta en las $48 \mathrm{hrs}$ posteriores a la administración de las terapéuticas. En la Monoterapia este síntoma posee $47 \%$ y por otro lado la Politerapia muestra $46 \%$.

Al comparar los datos de trismo de ingreso de cada grupo de estudio encontramos aproximación en los casos. Al inspeccionar el trismo de manera moderada se nota que en los pacientes que se ingresaron para manejo con politerapia están en 30\% de los casos. Sin embargo, en los de Monoterapia aparecen con un 35\%. En la información de los casos con trismo leve en la politerapia, se observa el 40\%; en los casos de Monoterapia se plasma un 45\% de los pacientes. Al verificar en la imagen gráfica, la politerapia presenta una adecuada apertura oral en 30\% de los casos y la Monoterapia revela un $20 \%$. Al comparar el trismo alas $48 \mathrm{hrs} \mathrm{de} \mathrm{terapéutica.} \mathrm{En} \mathrm{ambos} \mathrm{esquemas} \mathrm{de} \mathrm{tratamiento,} \mathrm{hay} \mathrm{un} \mathrm{semejanza}$ ya que al administrar Politerapia la adecuada apertura oral ocupo el $75 \%$ y en la Monoterapia un $30 \%$. 
En cambio el trismo presente de manera leve en la Politerapia ocurre en $25 \%$ y en la Monoterapia en $25 \%$.

Los días de estancia hospitalaria de los grupos de estudio donde en el tratamiento con Monoterapia se mostró que de 3 a 5 días ocupo el 75\% mientras en la Politerapia fue un 50\%. En intervalo de 6 a 10 días, ostentó en la Monoterapia en $25 \%$ y en la Politerapia 30\%. Además la politerapia presentó casos con intervalos de 11 a 15 días en un 15\% y en intervalos mayor a los 15 días con 5\% de los casos, sin embrago en la Monoterapia no existe casos reportados en este estudio con estos dos últimos intervalos de tiempo de estancia hospitalaria. Se muestra una diferencia al comparar ambos esquemas de tratamiento. Al ser administrada la politerapia observamos presencia de Nauseas con $25 \%$, mareos con $15 \%$ y trastornos gastrointestinales con $12.5 \%$ contrario de la Monoterapia que no presentó en la administración del tratamiento efectos adversos en su uso.

\section{DISCUSIÓN}

Las infecciones odontogénicas afectan por igual a la población en estudio no importando sexo, edad, escolaridad ni procedencia del paciente correspondiendo con los datos publicados por estudios internacionales de la Guías clínicas, edición española de la Guía Terapéutica Antimicrobiana (2006).

Con lo respecto a la eficacia del tratamiento con politerapia empleado en la terapéutica en contra de las infecciones odontogénicas. El empleo de la terapéutica con politerapia fue aplicado según las indicaciones internacionales de tratamiento para infecciones odontogénicas publicadas por the Oral and Maxillo facial Surgery Clinics of North America, el uso de la antibioticoterapia se llevó acabo con la adecuada posología, tanto la vía de administración, frecuencia y la dosis adecuada según las recomendaciones de la American Academy of Periodontology. La eficacia del empleo de la politerapia antibiótica, dado por la correcta aplicación del tratamiento mostró efectos adversos como mareos, náuseas y trastornos gastrointestinales. Esta terapéutica reveló, ser eficaz al disminuir en $48 \mathrm{hrs}$ los datos de leucocitosis y síntomas asociados a la infección odontogénica. Además de reducir el riesgo de compromiso de la vía aérea. En la mayoría de los casos de estudio poseían en su control hospitalario una adecuada apertura oral. Con el uso de la politerapia, los días de estancia hospitalaria para el control de las infecciones odontogénicas. A grandes rasgos, muestra que la duración oscila entre 5 y 10 días o, dicho de otra manera. El tratamiento se prolongó 3 o 6 días después de la desaparición de las manifestaciones clínicas de la infección.

Con respecto a la eficacia de la monoterapia en la terapéutica en contra de las infecciones odontogénicas. Esta terapéutica con Clindamicina se empleó en todos los pacientes tratados con 
monoterapia, en conjunto con eliminación del factor etiológico, manejo quirúrgico precoz y tratamiento de apoyo sistémico de soporte. Cumple los estándares de la American Academy of Periodontology. El antibiótico se administró con una adecuada vía, frecuencia y dosis de acuerdo a la guía Sanford to antimicrobial therapy, 2010. No se encontró en este estudio efectos adversos al aplicar esta terapéutica. Cuando se realizó el control de los pacientes en $48 \mathrm{hrs}$ de uso hospitalario de este tratamiento observamos que dicha terapia es eficaz en el control de la enfermedad. Valorado por la disminución de los síntomas clínicos, el conteo de los glóbulos blancos y por la presencia de una adecuada apertura oral de los pacientes. Los días de estancia hospitalaria de los pacientes de estudio con monoterapia, su mayoría fue entre 3 a 5 días. Lo que seguiré que el tratamiento duro entre 2 a 5 días, posteriores a la desaparición clínica de los síntomas. Relacionando las terapéuticas de manejo de las infecciones odontogénicas, se evidencia eficacia en ambos tratamientos de uso empírico que se administraron en los pacientes del estudio por el servicio de Maxilofacial. Los datos obtenidos en la comparación de las terapéuticas mostró que la Monoterapia es un tratamiento con menores afección hacia los pacientes con infecciones por no presentar efectos adversos, económicamente más rentable.

\section{CONCLUSIONES}

El tratamiento con politerapia posee eficacia contra las infecciones odontogénicas en pacientes con gravedad moderada. Pero implica un gasto mayor al hospital y menor tolerancia de los pacientes está terapéutica por poseer efectos adversos y mayores días de estancia hospitalaria. La monoterapia es eficaz para el manejo de infecciones odontogénicas de los pacientes con severidad moderada ya que posee un buen control de la enfermedad y sin presencia de reacciones adversas. La eficacia demostrada por la politerapia antibiótica contra la monoterapia aplicada de manera empírica a pacientes con infecciones odontogénicas. Presentan datos similares. Obteniendo de la administración de estos tratamientos presentando un adecuado control y erradicación de esta patología.

\section{REFERENCIAS}

Guía clínica, tratamiento afecciones orofaciales (Guía Sanford 2006). Guía Terapéutica en Atención Primaria Basada en la Evidencia. (2003) 